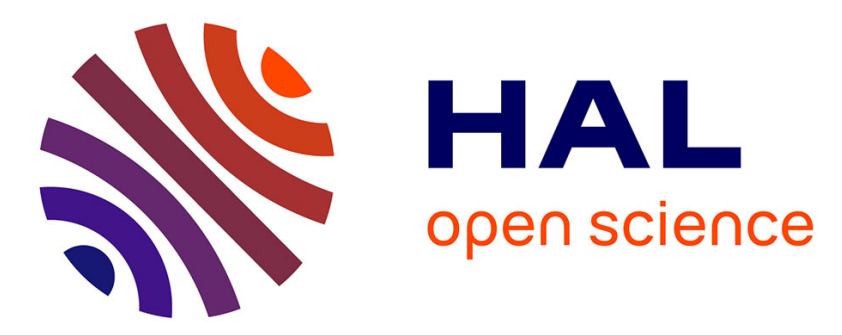

\title{
Modélisation de capteurs inductifs pour la mesure à travers une paroi métallique amagnétique
}

\author{
D. Placko, E. Santander
}

\section{To cite this version:}

D. Placko, E. Santander. Modélisation de capteurs inductifs pour la mesure à travers une paroi métallique amagnétique. Revue de Physique Appliquée, 1990, 25 (7), pp.721-735. 10.1051/rphysap:01990002507072100 . jpa-00246234

\section{HAL Id: jpa-00246234 https://hal.science/jpa-00246234}

Submitted on 1 Jan 1990

HAL is a multi-disciplinary open access archive for the deposit and dissemination of scientific research documents, whether they are published or not. The documents may come from teaching and research institutions in France or abroad, or from public or private research centers.
L'archive ouverte pluridisciplinaire HAL, est destinée au dépôt et à la diffusion de documents scientifiques de niveau recherche, publiés ou non, émanant des établissements d'enseignement et de recherche français ou étrangers, des laboratoires publics ou privés. 


\title{
Modélisation de capteurs inductifs pour la mesure à travers une paroi métallique amagnétique
}

\author{
D. Placko et E. Santander \\ L.E.SI.R., E.N.S. de Cachan, URA CNRS D1375, 61 Avenue du Président Wilson, 94235 Cachan Cedex, \\ France
}

(Reçu le 5 octobre 1989, révisé le 9 février 1990, accepté le 20 février 1990)

\begin{abstract}
Résumé. - Cet article présente une application originale utilisant les capteurs inductifs destinés à la mesure de déplacement d'une pièce magnétique à travers une paroi métallique amagnétique. Grâce à une analyse physique du problème, un modèle analytique permettant la prédétermination et l'optimisation des performances du système est élaboré, puis confronté avec succès aux résultats expérimentaux.
\end{abstract}

\begin{abstract}
In this paper, an interesting application of magnetic sensors used for distance measurement through a metallic medium is presented. By a careful physical analysis of this problem, an original modelling for predetermination and optimisation of the performances of this system is achieved, and successfully compared with experimental results.
\end{abstract}

\section{Introduction.}

L'automatisation actuelle des processus industriels nécessite l'implantation de capteurs destinés à fournir des informations locales assez précises portant sur un paramètre particulier (distance, épaisseur, position, vitesse, etc.).

Le choix du principe de mesure utilisé (optique, ultrasonore, magnétique...) dépend directement de la spécificité de l'application envisagée, et doit prendre en compte les problèmes d'encombrement, de portée, de résolution, mais aussi l'immunité aux perturbations environnantes, la durée de vie, etc.

Robustes et faciles à mettre en œuvre, les capteurs inductifs et à courants de Foucault (CCF) représentent une part importante des dispositifs utilisés. Leur principe est essentiellement lié à la mesure de la re uc ance un circul magne ique ouvert et parcouru par un flux magnétique alternatif. Ils sont sensibles à la modification de la répartition des lignes de champ magnétique provoquée par l'approche d'un objet métallique dans leur zone de perception.

Lorsque leurs qualités intrinsèques sont alliées, lors de la phase de conception, à une interprétation précise des signaux qu'ils délivrent, ces capteurs peuvent être employés à des applications beaucoup plus sophistiquées [1-3], élargissant le domaine classique qui leur est habituellement réservé (détection binaire de proximité, localisation de fissures, mesure de faibles déplacements).

Nous allons présenter ici une application assez particulière développée dans notre laboratoire, qui utilise des capteurs inductifs chargés de détecter le déplacement d'une cible au travers d'une paroi métallique amagnétique nécessaire au maintien de l'étanchéité d'une enceinte close [4].

La présence de ce milieu métallique complique autant la conception du système que l'interprétation des signaux fournis par le capteur qui dépendent fortement des paramètres électriques (conductivité et perméabilité) des divers matériaux (paroi, cible) traversés par le champ magnétique.

L'objet de cette publication consiste à décrire un modèle analytique de ce dispositif, suivi d'une phase lyse.

\section{Fonctionnement du dispositif.}

2.1 PRINCIPE. - Le circuit magnétique élémentaire est constitué d'un matériau à forte perméabilité en forme de « $U$ » muni d'un bobinage d'excitation ( $M$ spires) entourant le noyau central. Un plan métallique supposé infini, d'épaisseur $d$, le sépare de la cible (voir Fig. 1).

Le bobinage d'excitation parcouru par un courant 

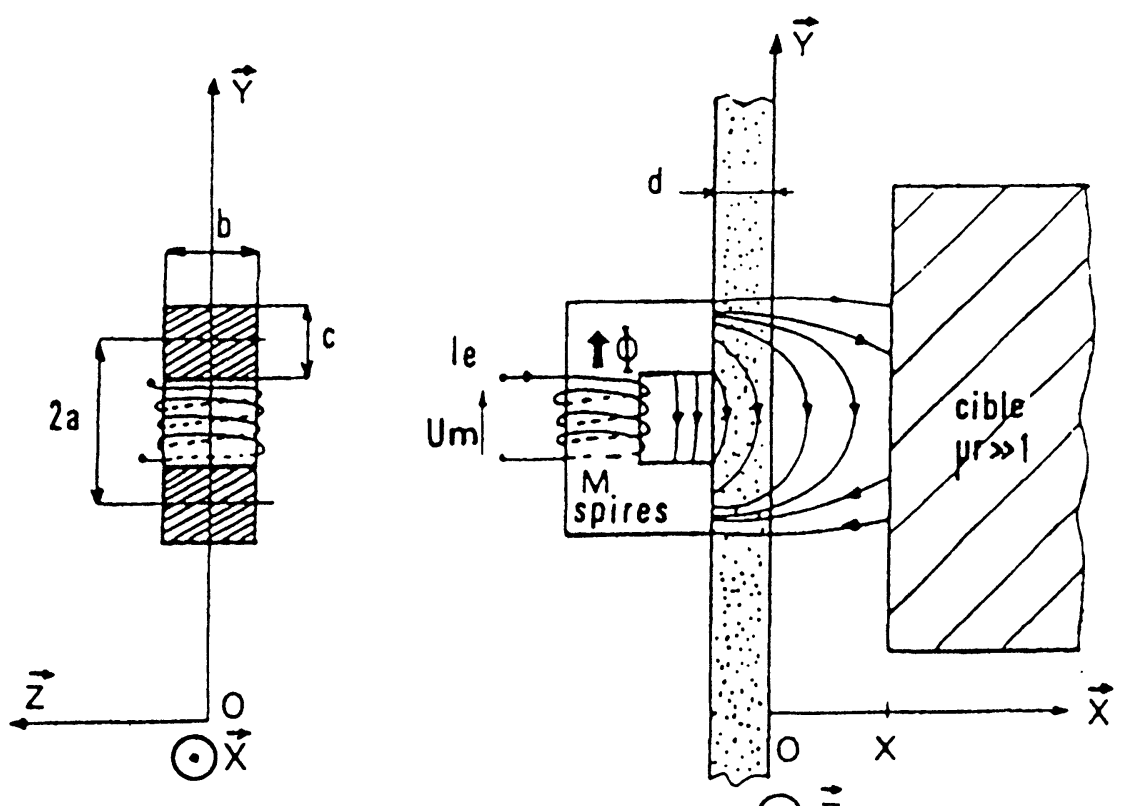

$\odot \vec{z}$

Fig. 1. - Circuit magnétique élémentaire.

[Basic magnetic circuit.]

sinusoïdal d'amplitude $I_{\mathrm{e}}$ et de fréquence $f$ impose une d.d.p. magnétique sous laquelle s'établit un flux $\Phi$ :

$$
M I_{\mathrm{e}}=R \Phi=\text { Cte } .
$$

$R$ étant la réluctance globale vue par le circuit magnétique ouvert.
La tension $U_{\mathrm{m}}$ induite aux bornes du bobinage (supposé sans fuites ni pertes) s'écrit alors :

$$
\begin{aligned}
U_{\mathrm{m}}=j M 2 \pi f \Phi=j 2 \pi f M^{2} I_{\mathrm{e}} / R & = \\
& =j 2 \pi f M^{2} I_{\mathrm{e}} G
\end{aligned}
$$

où $G$ représente la perméance égale à $1 / R$.

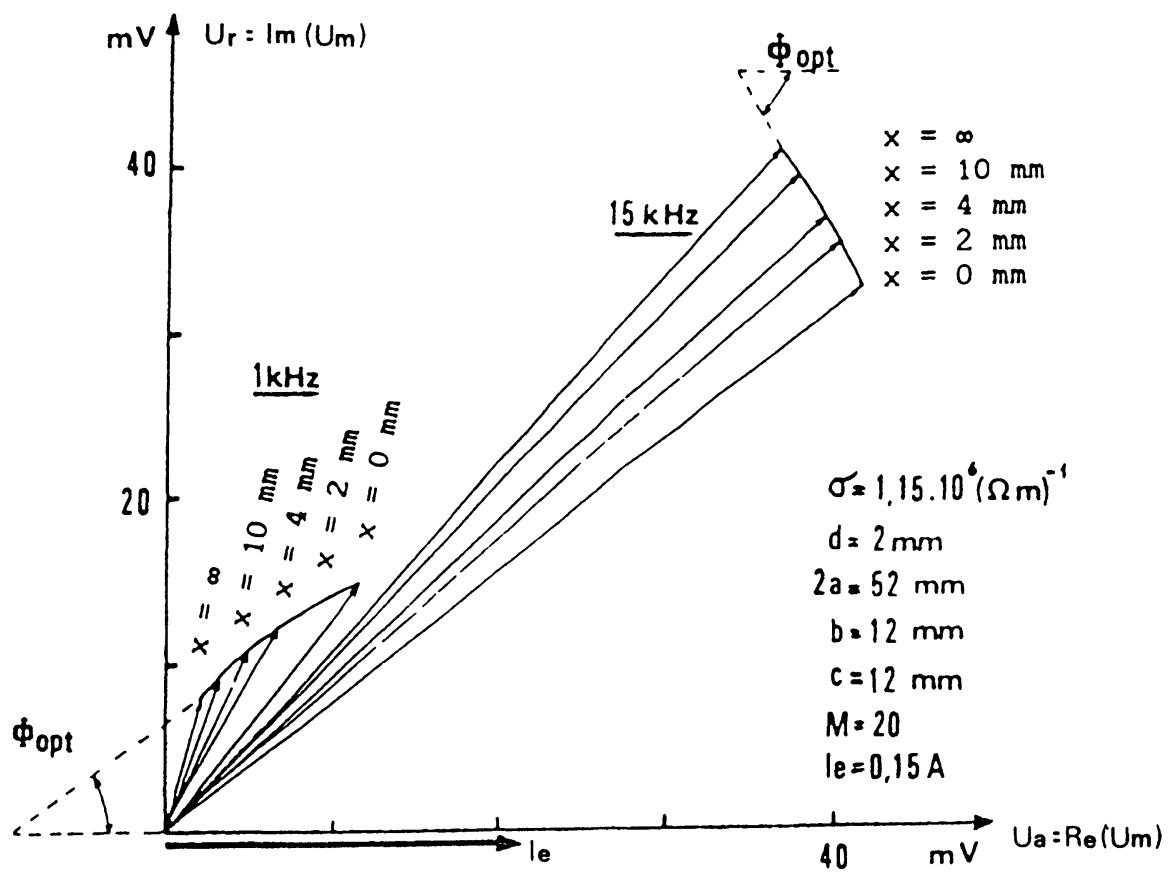

Fig. 2. - Diagramme des tensions capteur.

[Output signal vectors.] 
L'approche de la cible à forte perméabilité engendre une modification de la répartition des lignes de champ magnétique, ce qui entraîne une variation de la réluctance $R$ et donc du signal $U_{\mathrm{m}}$.

2.2 RÉPONSE DU CAPTEUR. - La présence de la paroi dans la zone d'émission du champ magnétique alternatif issu du capteur provoque l'apparition de courants induits dans le métal, appelés courants de Foucault. Le flux créé n'est alors plus en phase avec le courant d'excitation $I_{\mathrm{e}}$, ce qui peut s'interpréter aisément au niveau des équations précédentes en introduisant la notion de réluctance complexe. Dans ces conditions, le signal de sortie du capteur ne reflète plus un fonctionnement purement réactif car il comporte une partie réelle :

$$
U_{\mathrm{m}}=j U_{\mathrm{r}}+U_{\mathrm{a}} .
$$

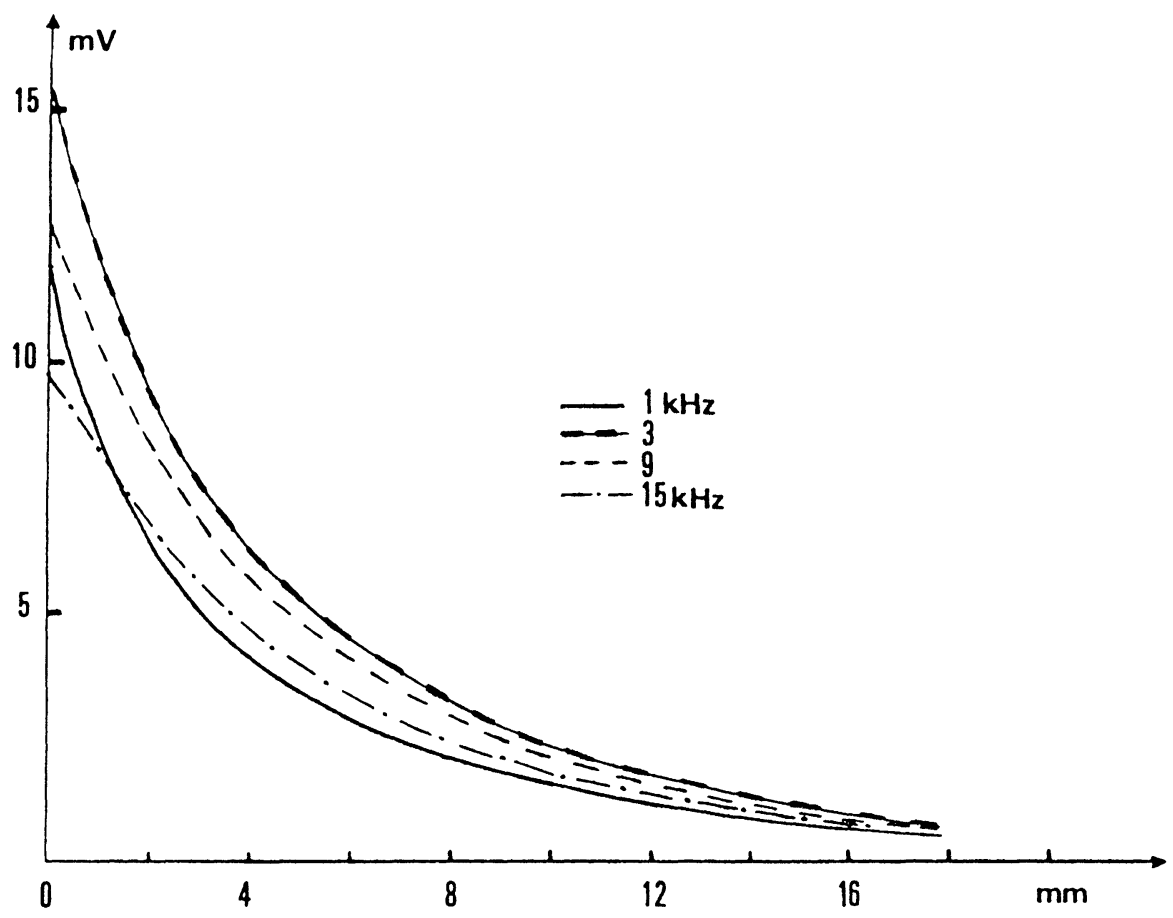

Fig. 3. - Variations du signal utile.

[Variations of the output signal with the distance.]

ont été relevées avec le même dispositif expérimental, pour différentes valeurs de la fréquence d'excitation; on constate la décroissance rapide du signal avec la distance, typique à ce genre de capteur.

L'interprétation de ces courbes, notamment en ce qui concerne l'influence des courants induits dans la paroi selon la fréquence d'excitation, est facilitée si on s'intéresse plutôt à la dynamique offerte par le système en fonction des divers paramètres géométriques et électriques qui le constituent. C'est une grandeur essentielle parce qu'elle peut être compa-
La figure 2 illustre les relations précédentes et présente le diagramme des tensions délivrées par un capteur selon l'éloignement progressif de la cible, pour deux fréquences d'excitation différentes. Les données géométriques et les propriétés électriques des éléments qui composent le système sont précisées sur la figure.

Remarquons que dans ce cas particulier, la portion de courbe sur laquelle évolue l'extrémité du vecteur "mesure " peut être assimilée à une droite formant un angle $\Phi_{\text {opt }}$ avec l'axe des abscisses. L'électronique de traitement du signal associée à ce capteur pourra exploiter simplement la valeur de cet angle $\Phi_{\text {opt }}$ au moyen d'une détection synchrone chargée d'extraire le terme utile à partir des signaux observés.

Les variations de ce signal utile en fonction de la distance qui sépare la paroi d'une cible magnétique plane parallèle sont données en figure 3 . Ces courbes 
fréquence pour un capteur identique à celui de la figure 2 :

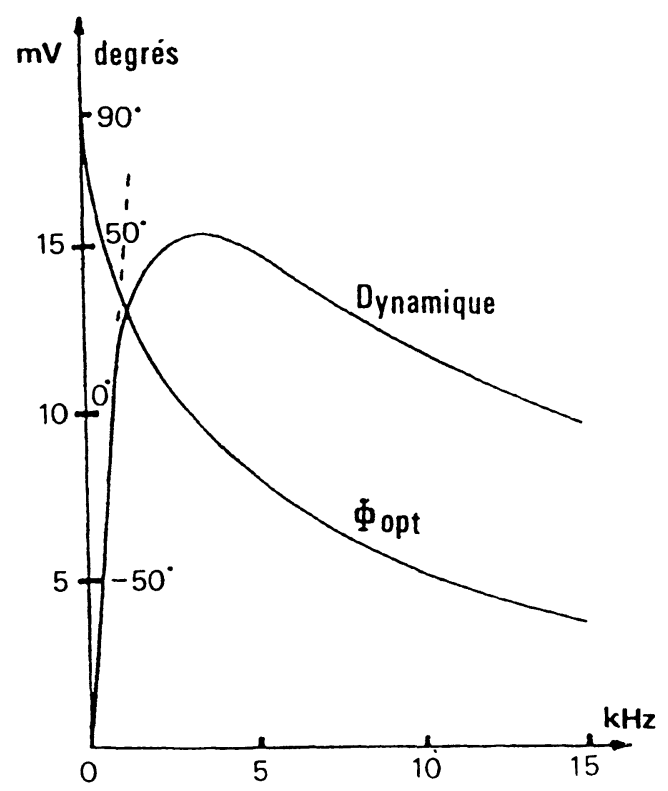

Fig. 4. - Dynamique et phase optimale en fonction de la fréquence.

[Dynamic range and optimal phase versus frequency.]

Aux fréquences basses pour lesquelles les courants de Foucault dans la paroi présentent une influence négligeable, le flux magnétique est quasiment en phase avec le courant d'excitation: le signal de sortie du capteur, $U_{\mathrm{m}}$, évolue alors de façon analogue à celui d'un capteur inductif classique $\left(U_{\mathrm{a}}=0\right)$ et croît directement avec la fréquence d'excitation. La phase optimale est alors proche de $90^{\circ}$.

Lorsque la fréquence augmente, l'ensemble des courants induits dans la paroi métallique constitue un obstacle important qui limite et déphase de plus en plus la fraction de flux capable d'atteindre la cible. Dans cette zone, l'évolution de la dynamique n'est plus proportionnelle à $f$ : au contraire, elle passe par un maximum, puis décroît. La position du maximum situé sur cette courbe permet de fixer une limite supérieure au domaine de fréquences dans lequel il est judicieux de faire fonctionner le capteur.

Afin de quantifier ces résultats nous exposons dans le prochain paragraphe un modèle analytique de notre dispositif.

Conduit à la base de certaines hypothèses originales, il va nous permettre d'obtenir des expressions simples à partir d'un problème électromagnétique complexe qui nécessiterait l'emploi d'une simulation numérique en 3D courants de Foucault. Pour le moment, ce type de code n'est pas opérationnel à $100 \%$, ce qui donne tout l'intérêt au développement de notre modèle.

\section{Modélisation.}

3.1 PRINCIPE DE L'ÉTUDE. - L'objet de cette étude consiste à faciliter l'interprétation des phénomènes observés lors de la phase expérimentale. Elle va nous permettre d'expliquer, par exemple l'existence d'une phase optimale, de quantifier la dynamique des signaux, mais aussi d'optimiser et de prévoir d'une manière générale le comportement de systèmes fonctionnant sur le principe exposé précédemment. L'originalité de notre modèle consiste à assimiler l'effet de la paroi interposé entre le capteur et la cible, à celui d'un quadripôle passif composé de réluctances complexes, interposé entre une source et une charge. Il nous permet, d'une part en calculant les éléments du quadripôle, et d'autre part en utilisant les notions de modèle dipolaire [5] et d'images électriques [6] pour représenter les variations de réluctance apportées par le déplacement de la cible, de déterminer la réluctance complexe vue par le capteur.

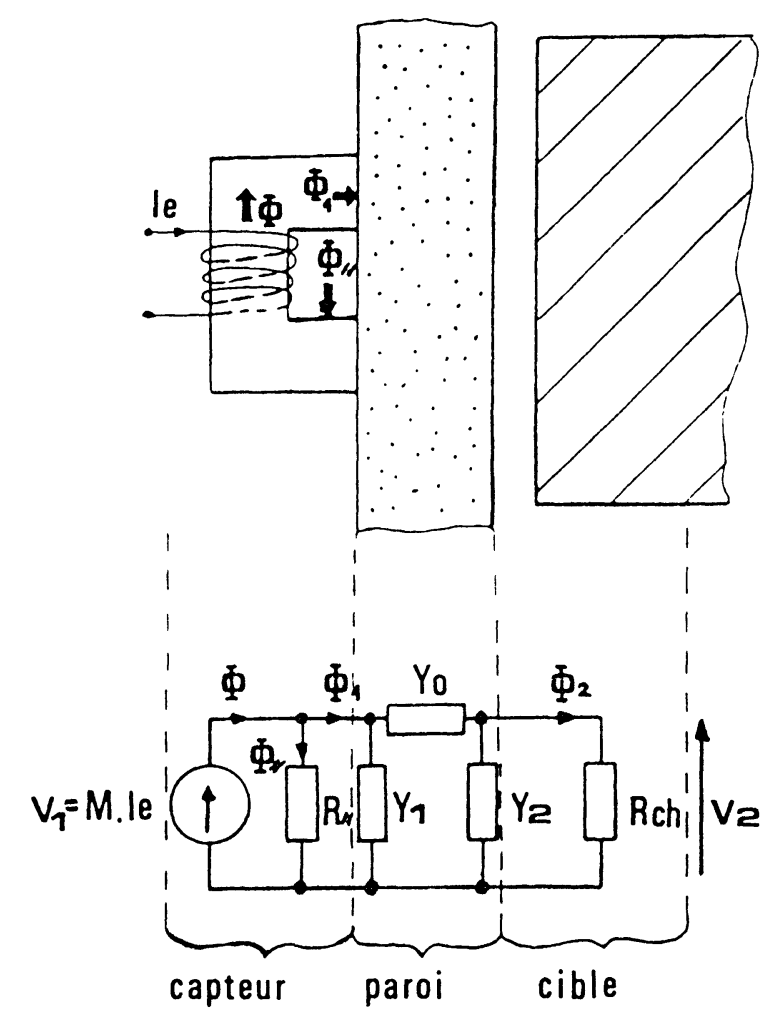

Fig. 5. - Modélisation par un quadripôle.

[Admittance matrix modelling.]

Un tel schéma est présenté en figure 5. Les éléments qui le composent prennent en considération les caractéristiques du capteur, représenté par une source à différence de potentiel magnétique constante, affectée d'une réluctance de fuite parallèle. 
Les relations entrée-sortie du quadripôle modélisant l'effet de la paroi peuvent être formulées selon une notation matricielle du type "perméance» :

$$
\left\{\begin{aligned}
\phi_{1} & =Y_{11} V_{1}+Y_{12} V_{2} \\
-\phi_{2} & =Y_{21} V_{1}+Y_{22} V_{2}
\end{aligned}\right.
$$

Le critère de réciprocité appliqué à ce quadripôle passif indique l'égalité des termes $Y_{12}$ et $Y_{21}$. D'autre part, il possède des propriétés de symétrie évidentes. L'identification avec la représentation de la figure 5 donne alors les égalités suivantes :

$$
\left\{\begin{array}{l}
Y_{1}=Y_{2}=Y_{11}-Y_{0} \\
Y_{0}=Y_{12}=Y_{21}
\end{array}\right.
$$

Le choix de la formulation "perméance" facilite, sur le plan expérimental, l'accès aux valeurs de $Y_{1}$ et $Y_{0}$ qui sont relevées "sortie en court-circuit" en mesurant les valeurs des flux $\phi_{1}$ et $\phi_{2}$ : il suffit d'amener un circuit magnétique à forte perméabilité en contact avec la face externe de la paroi et de le placer en vis-à-vis du capteur.

Le principal avantage de cette représentation consiste à pouvoir traiter indépendamment la modélisation de chaque élément.

3.2 SOURCE. - La d.d.p. magnétique de la source est donnée par l'expression :

$$
V_{1}=M I_{\mathrm{e}} .
$$

Du point de vue technologique, il est souhaitable de disposer d'un second bobinage exclusivement réservé à la mesure du flux afin de s'affranchir des chutes de tension dues aux pertes et fuites du bobinage d'excitation. En plaçant cet enroulement au voisinage immédiat de l'un des pôles, on mesure une part beaucoup plus faible du flux de fuite.

La réluctance de fuite parallèle $R_{\|}$(voir Fig. 4) est réduite à une réluctance $R_{\mathrm{f}}$, mesurée à l'aide d'une plaque en métal très conducteur (cuivre) placée directement contre les pôles du capteur. Celui-ci est excité à une fréquence suffisamment élevée pour rendre l'épaisseur de pénétration du champ électromagnétique dans ce métal négligeable vis-à-vis des autres dimensions géométriques du système. Cette démarche permet de s'approcher de l'hypothèse du " circuit ouvert ", nécessaire à cette mesure, puisque la réluctance vue par les pôles du capteur devient très élevée (voir Fig. 6).
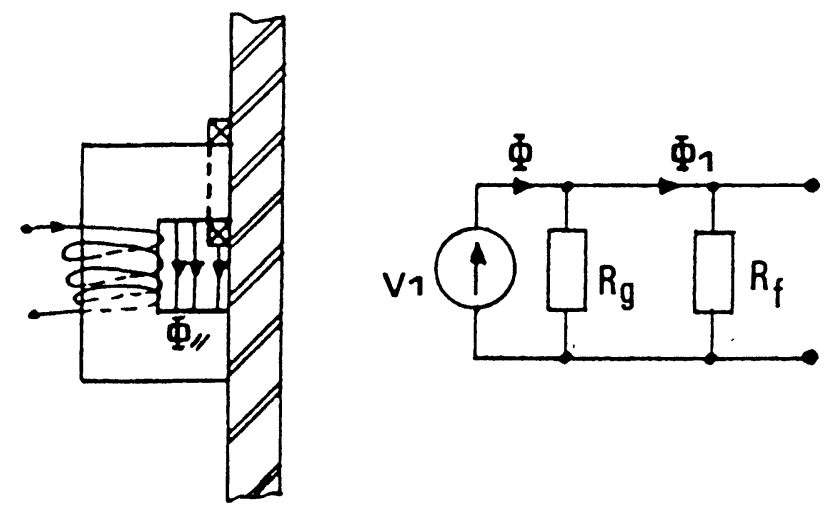

Fig. 6. - Mesure de l'élément $R_{\mathrm{f}}$ de la source.

[Measurement of the internal reluctance of the source.]

Ces conditions permettent la détermination de $R_{\mathrm{f}}$ :

$$
R_{\mathrm{f}}=\left(V_{1} / \Phi_{1}\right)
$$

Lors de cette mesure, il est nécessaire de considérer uniquement la partie réelle du flux mesuré, pour éliminer l'influence des pertes dans le cuivre, dont la conductivité n'est pas infinie ( $\Phi_{1}$ apparaît légèrement déphasé par rapport au courant $I_{\mathrm{e}}$ ).

3.3 ModélisAtion de LA CIBLE. - Les variations de réluctance provoquées par un déplacement (selon l'axe $x$ du capteur) de la cible plane parallèle à la paroi, sont étudiées sous certaines hypothèses.

D'une part, le matériau composant la cible est supposé infiniment perméable et non conducteur, ce qui permet dans une première étape, de lui substituer

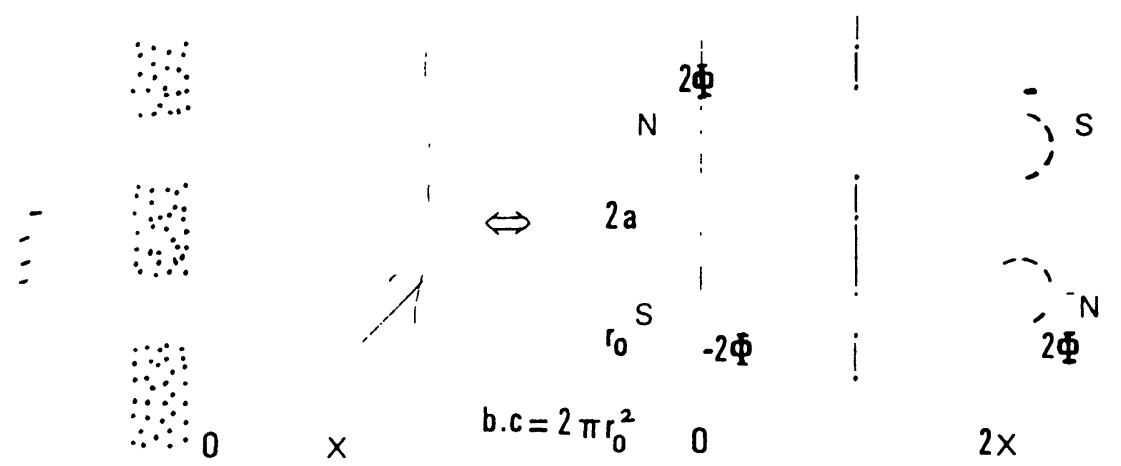

Fig. 7. - Modèle dipolaire.

[Dipolar model.] 
un "système image " (capteur plus paroi), disposé symétriquement par rapport à ce plan.

D'autre part, le champ émis par la paroi du côté cible est assimilé au champ qui serait émis par un dipôle magnétique présentant des caractéristiques géométriques voisines de celles du capteur (Fig. 7).

Cette modélisation conduit à des résultats analytiques simples, déjà développés et vérifiés lors de travaux précédents $[2,7]$. Nous présentons simplement ici les résultats essentiels :

$$
R_{\mathrm{ch}}=\mathcal{R}(x)=\mathcal{R}_{\infty}+\mathcal{R}_{i}(x)
$$

avec :

$$
\mathcal{R}_{\infty}=\frac{1}{\pi \mu_{0}}\left(\frac{1}{r_{0}}-\frac{1}{2 a-r_{0}}\right)
$$

et

$$
\begin{aligned}
& \mathcal{R}_{\mathrm{i}}(x)=-\frac{1}{\pi \mu_{0}} \times \\
& \times\left(\frac{1}{\left(4 x^{2}+r_{0}^{2}\right)^{1 / 2}}-\frac{1}{\left(4 x^{2}+\left(2 a-r_{0}\right)^{2}\right)^{1 / 2}}\right) .
\end{aligned}
$$

La validité de cette modélisation n'est obtenue que dans le cas d'une cible idéale, mais ces conditions sont quasiment vérifiées pour un matériau magnétique de bonne qualité. Toutefois, l'influence des propriétés réelles de ce matériau pourrait être quantifiée grâce à un modèle de faibles perturbations [3].

\subsection{DÉTERMINATION DES ÉLÉMENTS DE LA PAROI.}

3.4.1 Mesure de la perméance $Y_{0}$. - La détermination de cet élément nécessite la mesure du flux « en court-circuit » $\Phi_{2}$ (lorsque $V_{2}=0$ ). Pour cela nous avons placé en aval de la paroi un circuit magnétique muni d'un bobinage de mesure ( $N$ spires), formant un ensemble identique au capteur principal (voir Fig. 8).

La qualité du court-circuit ainsi réalisé peut être vérifiée en constatant que le flux dans le circuit principal $\Phi_{1}$ garde une valeur très voisine si on substitue au deuxième circuit magnétique une plaque de grandes dimensions constituée d'un matériau à forte perméabilité.
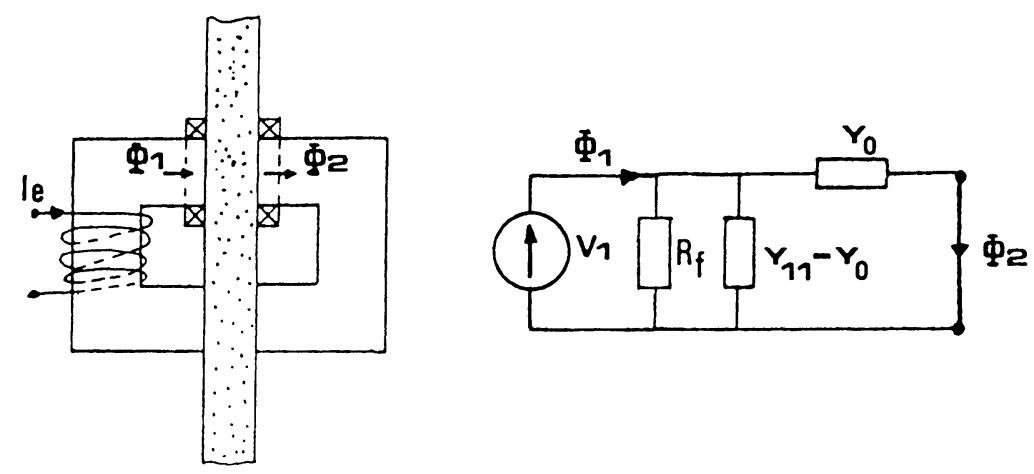

Fig. 8. - Mesure «en court-circuit» de $Y_{0}$.

[« Short-circuit» measurement for $Y_{0}$.]

La valeur de $Y_{0}$ s'obtient par:

$$
Y_{0}=\left(\Phi_{2} / V_{1}\right)_{V_{2}=0}
$$

3.4.2 Mesure de la perméance $Y_{1}=Y_{2}=Y_{11}-Y_{0}$. - La valeur de $Y_{0}$ étant prédéterminée, il nous suffit désormais de mesurer la valeur de $Y_{11}$ pour en déduire celle de $Y_{1}=Y_{2}$. Celle-ci s'obtient également par le court-circuit de la figure 8 en relevant cette fois-ci la valeur du flux $\Phi_{1}$ :

$$
Y_{11}=\left(\Phi_{1} / V_{1}\right)_{V_{2}=0}-1 / R_{\mathrm{f}}
$$

3.5 VALIDATION DU MODÈLE QUADRIPÔLE. Pour valider les hypothèses effectuées lors de la modélisation précédente, nous avons calculé l'évolution de la réluctance complexe ramenée à l'entrée (à l'aide du schéma de la Fig. 5), lorsque la réluctance de charge varie. Nous insérons les éléments «mesurés » pour les impédances composant le quadripôle et la source, et utilisons la modélisation de la charge développée plus haut.

Les courbes présentées figure 9 ont été relevées pour un système dont la topologie est identique à celui de la figure 2 . Elles permettent d'établir une comparaison favorable entre les données expérimentales (traits continus) et les valeurs calculées par le modèle (traits pointillés), qu'il s'agisse de la partie réelle ( $\mathrm{Re})$ ou de la partie imaginaire (Im) de «l'impédance » vue par le capteur.

\subsection{MOdÉlisATION DES ÉLÉMENTS DE LA PAROI.}

3.6.1 Hypothèses. - Nous avons déterminé précédemment les différents éléments de la paroi par des 

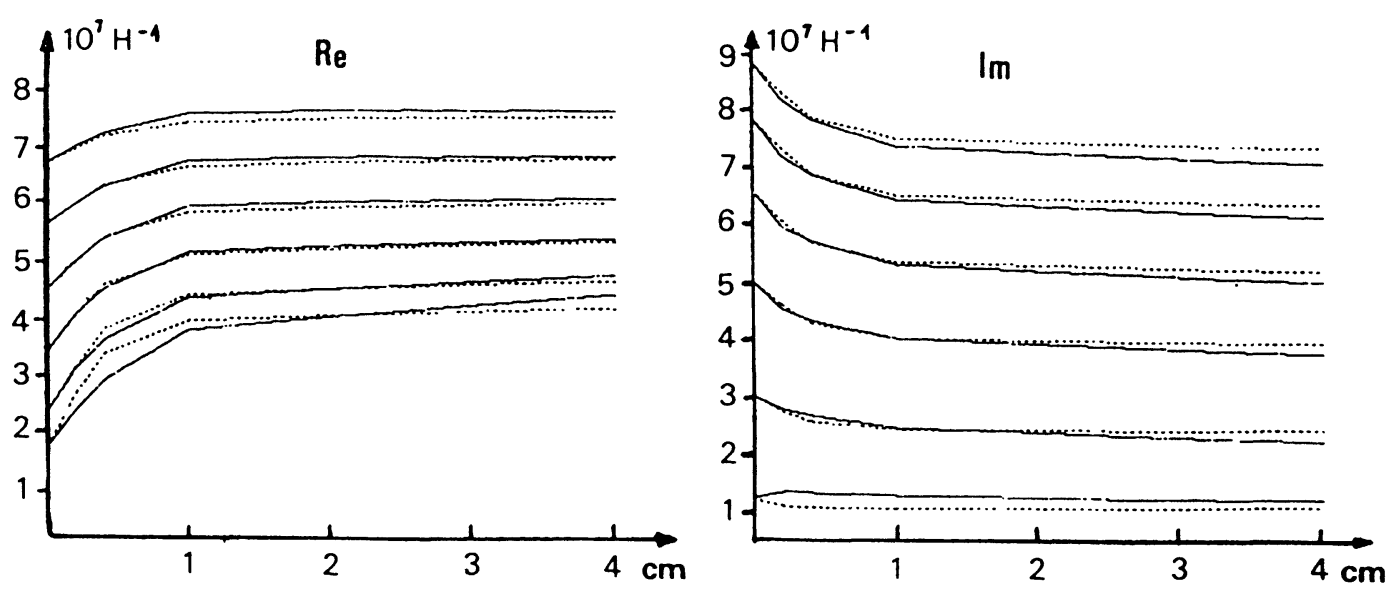

Fig. 9. - Validation du modèle quadripôle pour la paroi.

[Validity of the matrix model.]

mesures « en court-circuit ». Dans ces conditions, les lignes de champ magnétique émises par les pôles sont considérablement attirées vers la matière magnétique et les tubes de flux ainsi formés présentent une divergence assez faible, comme l'indique la simulation numérique 2D courants de Foucault présentée figure $10 \mathrm{a}$.

Les résultats satisfaisants obtenus précédemment grâce à notre "modèle d'observation " montrent que les valeurs des éléments du quadripôle relevés dans de telles conditions peuvent être conservés pour rendre compte du fonctionnement du système lorsque la distance entre la cible et la paroi devient importante, ce qui signifie que la topologie des lignes de champ n'est pas trop affectée par la variation de ce paramètre. Les figures $10 \mathrm{~b}$ et $10 \mathrm{c}$ présentent les résultats de la simulation numérique lorsque la distance est fixée respectivement à 2 et $4 \mathrm{~mm}$, pour deux fréquences d'excitation différentes.

A partir de l'ensemble de ces résultats, nous allons développer l'idée selon laquelle l'effet de la paroi sur le flux émis par le capteur peut être représenté par les éléments d'une ligne bifilaire à constantes réparties, en supposant invariantes les données géométriques d'une tranche infiniment mince de cette « ligne », quel que soit l'emplacement qu'elle occupe

Il suffira ensuite de déterminer le quadripôle équivalent à la ligne bifilaire totale pour obtenir un modèle analytique des termes $Y_{0}$ et $Y_{1}$ déjà évoqués, qui représenteront respectivement les perméances situées sur le trajet « longitudinal » et « transversal » du flux magnétique. Un ajustement des paramètres géométriques de cette ligne sera éventuellement utile pour tenir compte de l'épanouissement réel des lignes de champ émises par les pôles du capteur.

3.6.2 Elément série de la ligne bifilaire. - Cet élément est calculé à partir de la chute de potentiel magnétique existant dans un élément de paroi $\mathrm{dx}$ parcouru par les flux longitudinaux $\Phi$ et $-\Phi$.

3.6.2.1 Champ à l'extérieur du tube de flux magnétique. - Nous allons considérer un seul tube de flux compte tenu de la géométrie du problème, et nous adoptons le système de coordonnées cylindriques de la figure 11 .

L'utilisation de l'équation de Maxwell $\operatorname{rot} \mathbf{E}=$ $-\partial \mathbf{B} / \partial t$ appliquée à un champ électromagnétique harmonique nous amène à écrire pour un milieu homogène, isotrope $(\sigma, \mu)$ :

$\operatorname{rot} \mathbf{E}=-j \omega \mathbf{B} \Rightarrow \int \mathbf{E} \cdot \mathrm{dI}=-j \omega \iint \mathbf{B} \cdot \mathrm{d} \mathbf{S}$.

Imposons au terme $\iint \mathbf{B} . \mathrm{d} \mathbf{S}$ la valeur $\phi$ dans tout l'espace où $\rho \geqslant r$, nous obtenons :

$$
E_{\mathrm{e}}(\rho)=\frac{j \omega \Phi}{2 \pi \rho} .
$$

La chute linéique de potentiel créée par la somme des courants situés dans la tranche $\mathrm{d} x$ entre $r$ et $r_{\text {ext }}$ est donnée par l'expression suivante :

$$
-\frac{\mathrm{d} V_{\mathrm{e}}}{\mathrm{d} x}=\int_{r}^{r_{\mathrm{ext}}} \sigma E_{\mathrm{e}} \cdot \mathrm{d} \rho=\left[H_{\mathrm{e}}(\rho)\right]_{r}^{r_{\mathrm{ext}}}
$$

avec :

$$
H_{\mathrm{e}}(\rho)=\frac{j \sigma \omega \Phi}{2 \pi} \ln \rho
$$

La chute de potentiel ainsi créée est équivalente à celle que présenterait un anneau métallique très mince de diamètre extérieur $r_{\text {ext }}$, intérieur $r$, traversé en son centre par un flux sinusoïdal d'amplitude $\phi$. 

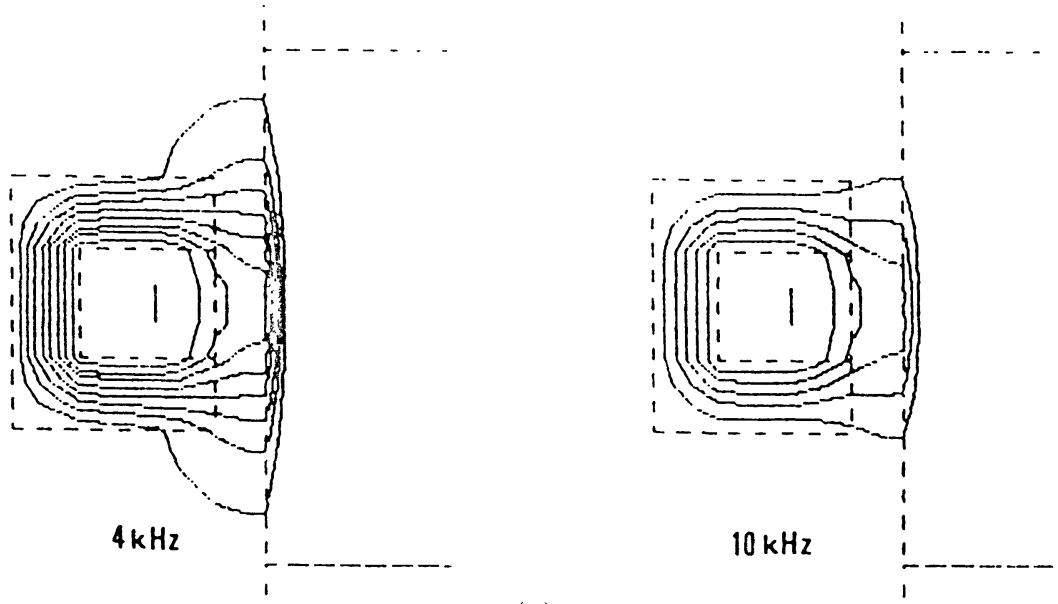

(a)
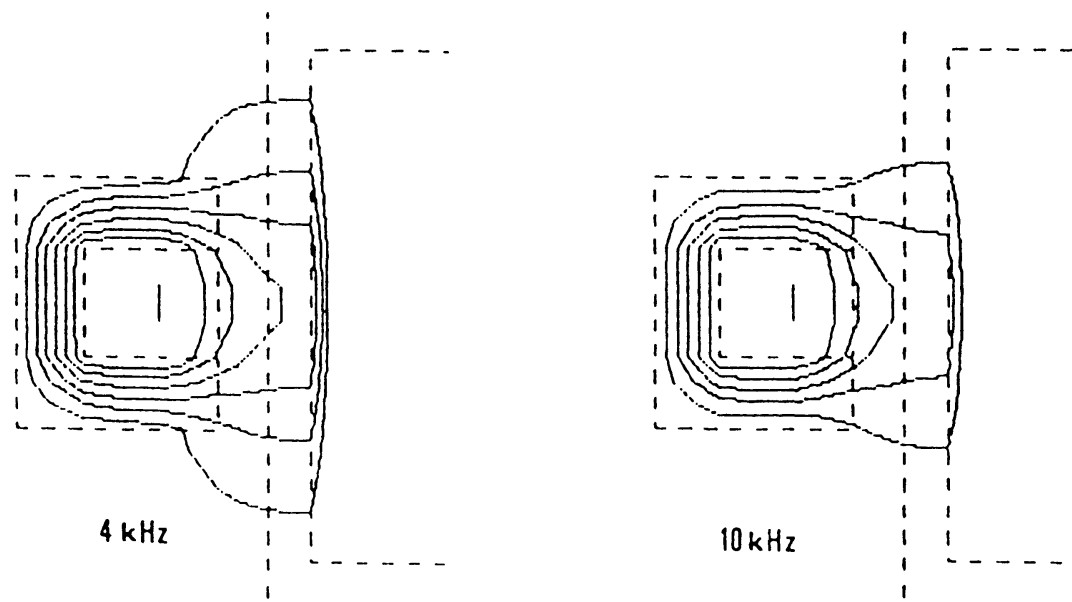

(b)
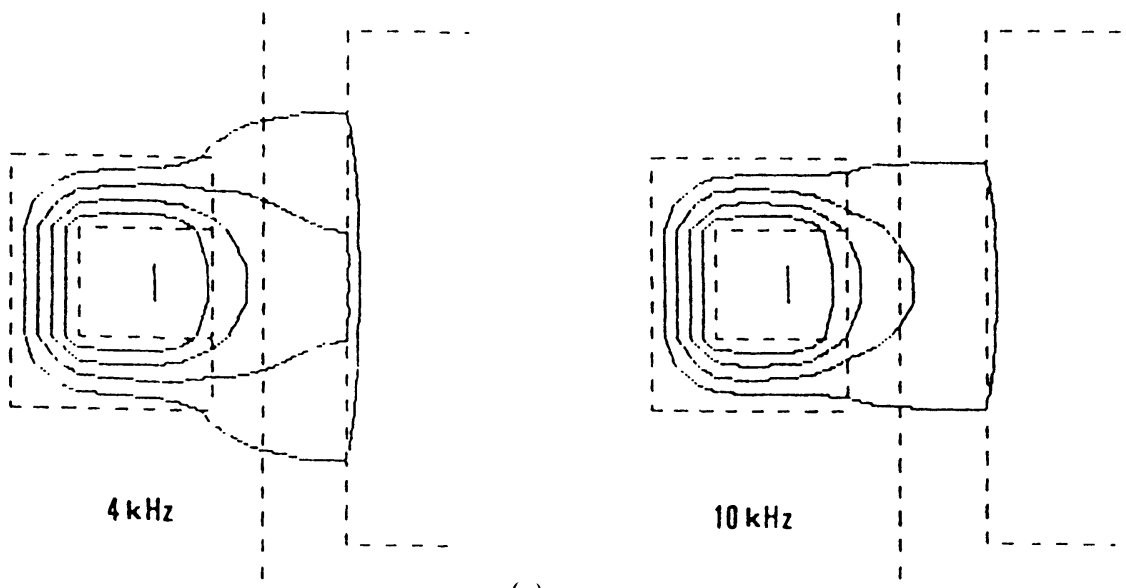

(c)

Fig. 10. - a) Répartition des lignes de champ pour $x=0$. b) Répartition des lignes de champ pour $x=2 \mathrm{~mm}$. c) Répartition des lignes de champ pour $x=4 \mathrm{~mm}$.

[a) Magnetic field path for $x=0$. b) Magnetic field path for $x=2 \mathrm{~mm}$. c) Magnetic field path for $x=4 \mathrm{~mm}$.] 

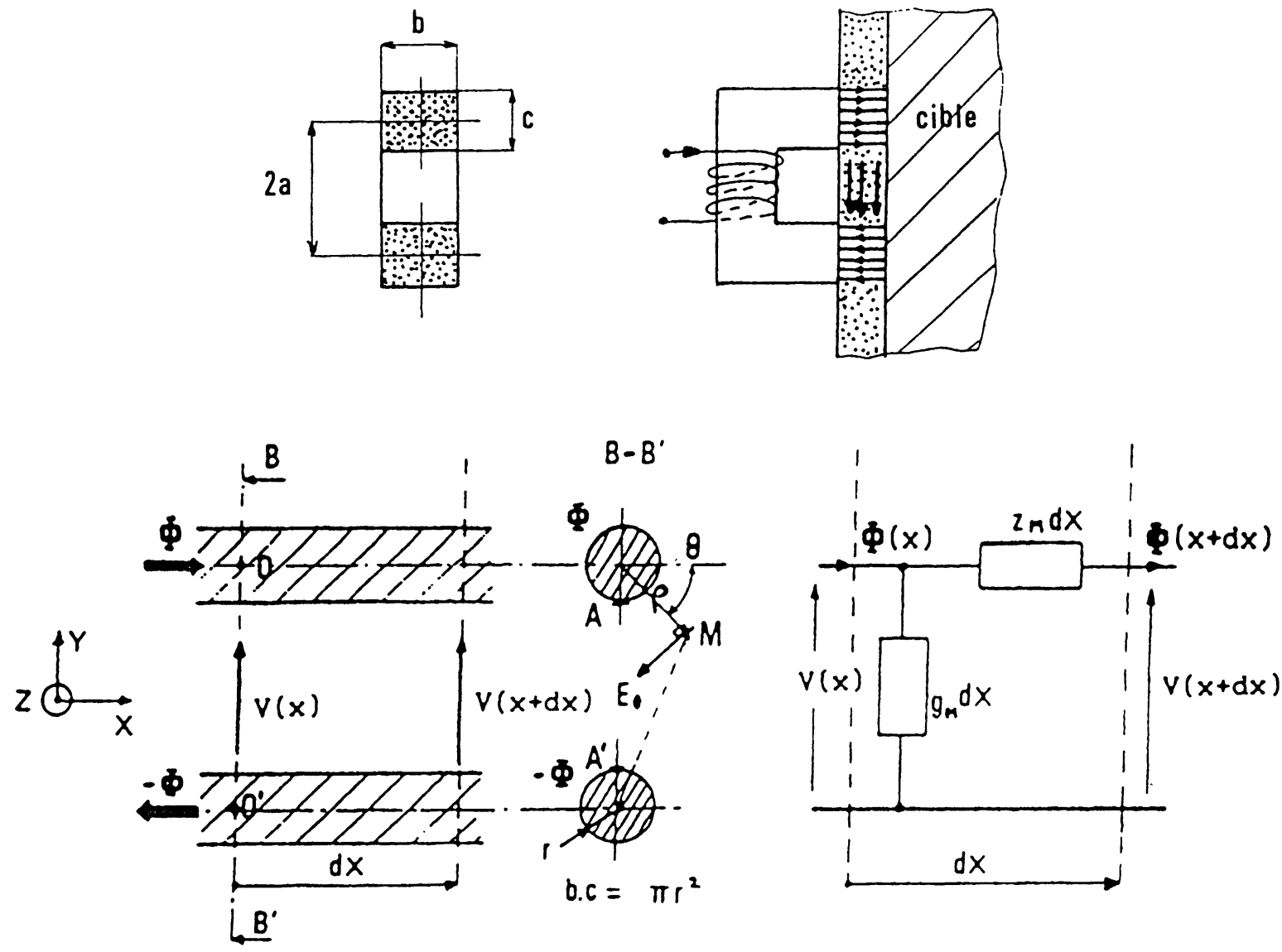

Fig. 11. - Ligne bifilaire équivalente.

[Equivalent twin conductors line.]

Cette configuration est d'ailleurs comparable à celle d'un transformateur dont le secondaire est constitué d'une spire en court-circuit. L'hypothèse simplificatrice consiste à supposer que l'ensemble du flux créé par les courants induits dans cette "spire » s'établit dans le circuit principal, ce qui revient à négliger l'effet de son flux (donc son inductance) de fuite vis-à-vis de sa résistance interne.

Il a été aisé, d'ailleurs, de vérifier la validité de l'équation (15) au moyen d'un circuit magnétique fermé comprenant un bobinage d'excitation et un secondaire constitué d'un anneau mince en cuivre, en faisant varier son diamètre extérieur.

que. - Dans cette zone, il est possible d'intégrer sans faire d'approximations les équations de Maxwell pour déterminer de façon plus complète l'expression du champ $\mathbf{E}$ et prendre en considération les courants induits qui s'y créent.

Il faut résoudre l'équation :

$$
\nabla^{2} \mathbf{E}=j \omega \sigma \mu \mathbf{E} .
$$

Le champ électrique $\mathbf{E}$ possède une seule composante suivant $\theta$ qui dépend uniquement de la variable $\rho$. L'équation (16) devient :

$$
\frac{\partial^{2} E_{\mathrm{i}}}{\partial \rho}+\frac{1}{\rho} \frac{\partial E_{\mathrm{i}}}{\partial \rho}--\left(j \omega \sigma \mu+\frac{1}{\rho^{2}}\right) E_{\mathrm{i}}=0 .
$$

La solution recherchée doit être finie pour $\rho=0$; par conséquent la solution de cette équation est une fonction de Bessel de première espèce d'ordre 1 à argument complexe [8,9]. En imposant le flux $\Phi$ en $\rho=r$ on obtient :

$$
E_{\mathrm{i}}(\rho)=\frac{j \omega \Phi}{2 \pi r} \frac{J_{1}[(j-1) \rho / \delta]}{J_{1}[(j-1) r / \delta]} .
$$

La relation donnant la chute linéique de potentiel s'écrit :

$$
-\frac{\mathrm{d} V_{\mathrm{i}}}{\mathrm{d} x}=\int_{0}^{r} \sigma E_{\mathrm{i}} \cdot \mathrm{d} \rho=\left[H_{\mathrm{i}}(\rho)\right]_{0}^{r}
$$

avec :

$$
H_{\mathrm{i}}(\rho)=\frac{(j-1) \Phi}{\mu 2 \pi r \delta} \frac{J_{0}[(j-1) \rho / \delta]}{J_{1}[(j-1) r / \delta]} .
$$

3.6.2.3 Calcul du terme linéique série. - Le potentiel magnétique linéique entre les points $O$ et $P$ de la figure 12 est obtenu par :

$$
-\frac{\mathrm{d} V_{\mathrm{M}}}{\mathrm{d} x}=\left[H_{\mathrm{i}}\right]_{0}^{r}+\left[H_{\mathrm{e}}\right]_{r}^{r_{\mathrm{ext}}} .
$$




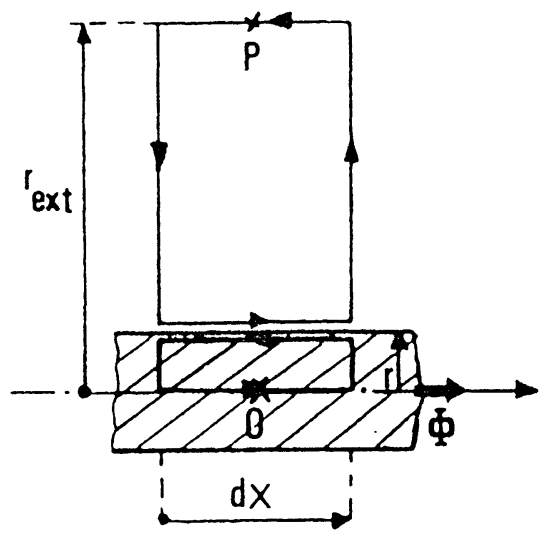

Fig. 12. - Potentiel entre $O$ et $P$ pour un seul tube de flux.

[Magnetic potential between $O$ and P.]

Dans le cas des deux tubes de flux distants de $2 a$ on a par superposition :

$$
-\frac{\mathrm{d} V_{\mathrm{M}}}{\mathrm{d} x}=2\left[H_{\mathrm{i}}\right]_{0}^{r}+2\left[H_{\mathrm{e}}\right]_{r}^{2 a}=\lambda_{\mathrm{M}} \Phi
$$

avec

$$
\begin{aligned}
& \lambda_{\mathrm{M}}=\frac{(j-1)\left(-1+J_{0}[(j-1) r / \delta]\right)}{\mu \pi r \delta J_{1}[(j-1) r / \delta]}+ \\
&+\frac{j \sigma \omega}{\pi} \ln \frac{2 a}{r} .
\end{aligned}
$$

Ce terme représente uniquement l'effet des courants induits dans le métal; on vérifie aisément qu'il tend vers zéro quand la pulsation d'excitation s'annule. Le champ magnétique interne $H_{\mathrm{i}}$ devient alors :

$$
\lim _{\omega \rightarrow 0} H_{\mathrm{i}}(\rho)=\frac{\Phi}{\mu \pi r^{2}}
$$

La circulation de ce champ le long du trajet $\mathrm{dx}$ pour les deux tubes de flux donne la réluctance linéique "statique »:

$$
r_{\mathrm{M}}=\frac{2}{\mu \pi r^{2}} .
$$

L'élément série de la ligne bifilaire est finalement :

$$
z_{\mathrm{M}}=r_{\mathrm{M}}+\lambda_{\mathrm{M}} \text {. }
$$

Remarquons que $\lambda_{M}$ est un terme complexe approximativement égal à $\frac{j \sigma \omega}{\pi} \ln \frac{2 a}{r}$ lorsque le rapport $r / \delta$ est petit devant 1 . De plus, lorsque l'épaisseur (d) de la paroi est faible, il est possible d'écrire :

$$
Z_{0} \cong z_{\mathrm{M}} \cdot d .
$$

Dans ces conditions, on constate que l'élément « série " $Z_{0}=1 / Y_{0}$ de notre quadripôle possède une partie réelle constante, ainsi qu'une partie imaginaire proportionnelle à la fréquence. Ceci justifie partiellement l'existence de la "barrière" qui limite la fraction du flux capable d'atteindre la cible lorsque la fréquence augmente.

3.6.3 Elément parallèle de la ligne bifilaire. - Pour calculer cet élément nous devons établir la relation qui existe entre le potentiel magnétique à une abscisse $x$ donnée et la diminution du flux longitudinal provoquée par le flux de fuite transversal. L'élément de ligne $\mathrm{d} x$ est infiniment petit, aussi l'influence des courants induits par le flux transversal peut être considérée comme négligeable.

Considérons la coupe B-B' de la figure 11 et appelons $Q$ et $-Q$ les flux magnétiques linéiques obtenus. Le potentiel magnétique créé en un point $M$ est la somme des potentiels magnétiques créés indépendamment par les masses magnétiques $Q$ et $-Q$, le résultat est classique et s'écrit :

$$
V(M)=\frac{Q}{2 \pi \mu} \ln \frac{\mathrm{O}^{\prime} \mathrm{M}}{\mathrm{OM}} .
$$

Le potentiel existant entre les points $A$ et $\mathrm{A}^{\prime}$ de la ligne bifilaire s'exprime donc par:

$$
\begin{aligned}
V_{\mathrm{M}} & =V(\mathrm{~A})-V\left(\mathrm{~A}^{\prime}\right) \\
& =\frac{Q}{2 \pi \mu}\left(\ln \frac{\mathrm{O}^{\prime} \mathrm{A}}{\mathrm{OA}}-\ln \frac{\mathrm{O}^{\prime} \mathrm{A}^{\prime}}{\mathrm{OA}^{\prime}}\right) \\
V_{\mathrm{M}} & =\frac{Q}{\pi \mu} \ln \left(\frac{2 a-r}{r}\right) .
\end{aligned}
$$

On peut alors définir la perméance linéique parallèle de la ligne :

$-\frac{\mathrm{d} \Phi}{\mathrm{d} x}=g_{\mathrm{M}} \mathrm{V}_{\mathrm{M}}$ avec $g_{\mathrm{M}}=\pi \mu / \ln \left[\frac{2 a-r}{r}\right]$.

3.6.4 Quadripôle équivalent. - Pour achever la phase de modélisation, il nous faut maintenant calculer le quadripôle équivalent à la ligne bifilaire totale. Nous allons déterminer la d.d.p. et le flux magnétique à la sortie en fonction des grandeurs correspondantes à l'entrée. Ce calcul est classique, nous allons énoncer ici seulement les résultats obtenus [10].

En définissant $Z_{\mathrm{c}}=1 / Y_{\mathrm{c}}$ comme l'impédance caractéristique de la ligne, nous avons les relations suivantes :

$$
\left\{\begin{array}{c}
V_{2}=\operatorname{ch}(\alpha d) V_{1}-Z_{\mathrm{c}} \operatorname{sh}(\alpha d) \Phi_{1} \\
-\Phi_{2}=-Y_{\mathrm{c}} \operatorname{sh}(\alpha d) V_{1}+\operatorname{sh}(\alpha d) \Phi_{1}
\end{array}\right.
$$

avec

$$
\alpha=\left(g_{\mathrm{M}} z_{\mathrm{M}}\right)^{1 / 2} \text { et } Z_{\mathrm{c}}=1 / Y_{\mathrm{c}}=\left(z_{\mathrm{M}} / g_{\mathrm{M}}\right)^{1 / 2} \text {. }
$$

Cette équation nous permet de calculer facilement les éléments du modèle formulé avec une matrice $Y$, défini aux paragraphes 3.1 et 3.4. On obtient: 


$$
\begin{gathered}
Y_{0}=\left(-\Phi_{2} / V_{1}\right)_{V_{2}=0}=Y_{\mathrm{c}} / \operatorname{sh}(\alpha d) \\
Y_{11}=\left(\Phi_{1} / V_{1}\right)_{V_{2}=0}=Y_{\mathrm{c}} / \operatorname{th}(\alpha d) \\
Y_{2}=Y_{11}-Y_{0}= \\
=Y_{\mathrm{c}}(\operatorname{ch}(\alpha d)-1) / \operatorname{sh}(\alpha d) \\
=Y_{0}(\operatorname{ch}(\alpha d)-1) .
\end{gathered}
$$$$
Y_{1}=Y_{2}=Y_{11}-Y_{0}=
$$

(31) tion (26) énoncé au paragraphe 3.6.2.3. On constatera également que $Y_{1}$ tend vers une valeur très faible.

\section{Confrontation théorique et expérimentale.}

Pour des parois minces $\alpha d$ est petit et un développement limité au premier ordre des fonctions hyperboliques permet de retrouver le résultat de l'équa-
4.1 ElÉment $Z_{0}=1 / Y_{0}$. - Les courbes expérimentales (traits continus) et théoriques (traits pointillés) de cet élément sont présentées en fonction de la fréquence sur la figure 13 dans le plan complexe pour trois capteurs et trois épaisseurs différentes.
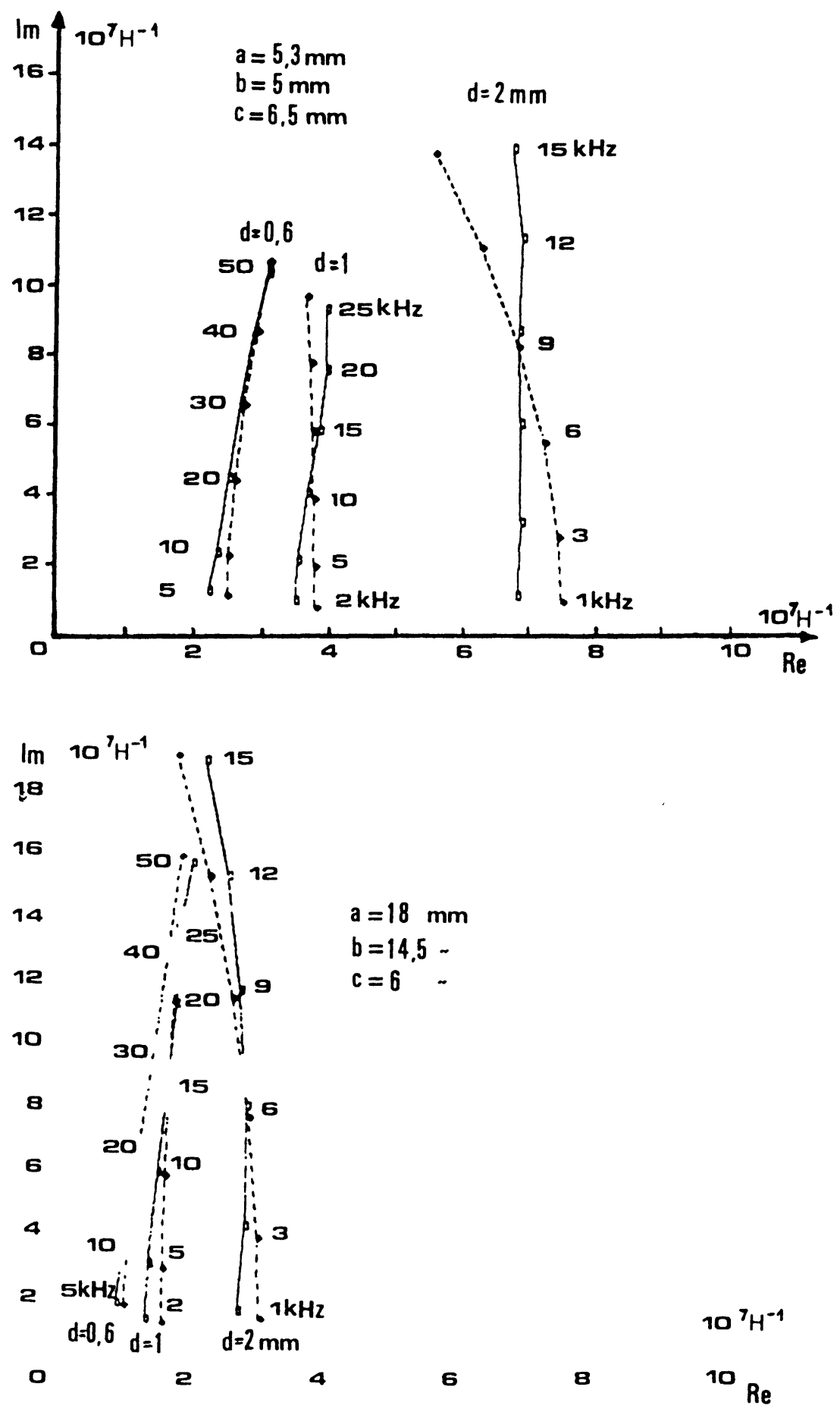

Fig. 13. 


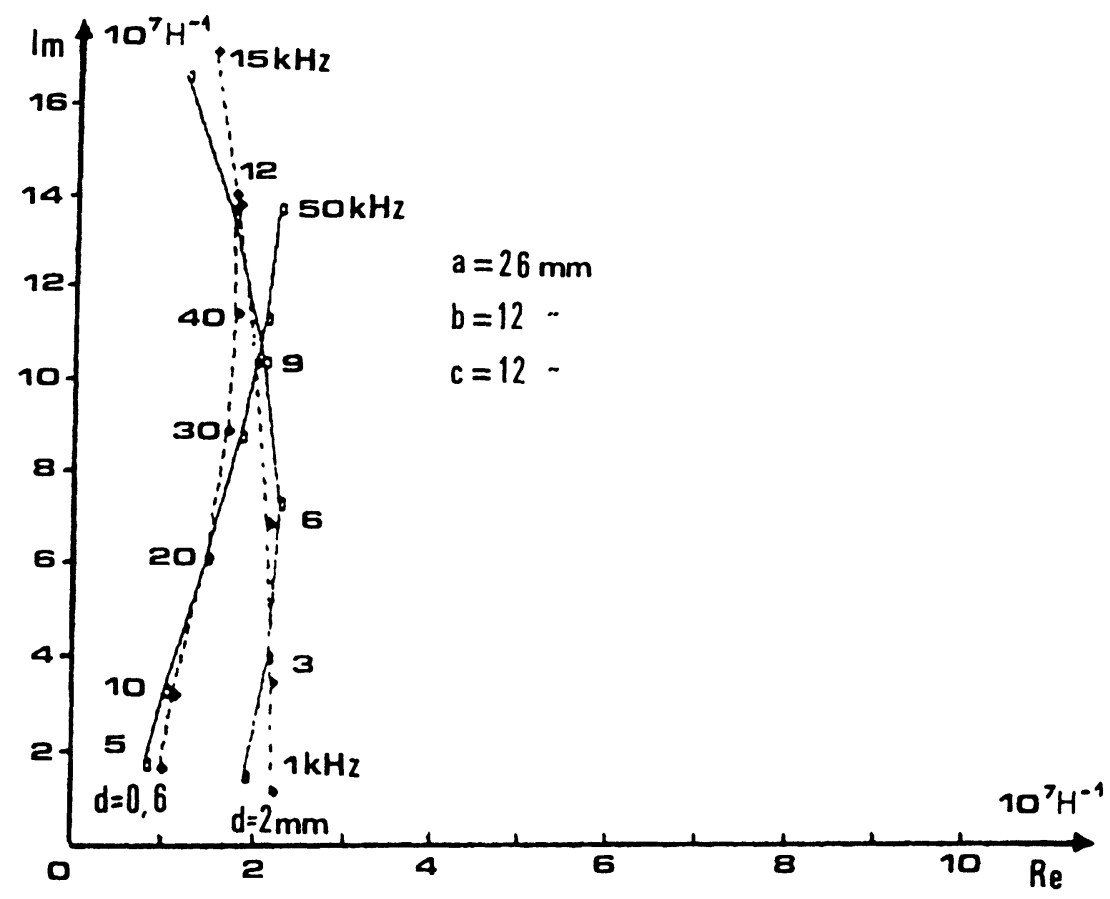

Fig. 13. - Variations du terme $Z_{0}$.

$\left[Z_{0}\right.$ variations.]

La bonne concordance entre ces courbes est obtenue grâce à un ajustement des paramètres $a$ et $r$ des tubes de flux magnétiques. Celui-ci se justifie compte tenu de l'hypothèse simplificatrice faite sur la répartition du champ magnétique dans la paroi : en réalité, les lignes de champ s'épanouissent naturellement en présence d'un entrefer et ne forment pas un tube de flux de rayon constant (voir Fig. 14).

Il est d'ailleurs probable, comme les simulations numériques le laissent supposer, que les lignes de champ s'épanouissent plus volontiers vers l'extérieur du capteur que dans la zone de la paroi située entre les deux pôles, compte tenu du champ antagoniste créé à l'intérieur de ce volume dans lequel les densités de courants induits sont les plus élevées. Cet effet est amplifié lorsque l'épaisseur de la paroi augmente et il est alors plus difficile d'ajuster le couple $(a, r)$ pour se rapprocher des courbes théoriques.

4.2 ELÉMENT $Z_{1}=1 / Y_{1}$. - Les valeurs expérimentales de ce terme sont faibles vis-à-vis des autres éléments constituant le modèle. Par conséquent, les mesures sont délicates et peuvent être entachées d'erreurs ; elles proviennent essentiellement des flux de fuite "parasites " détectés par les bobinages de mesure.

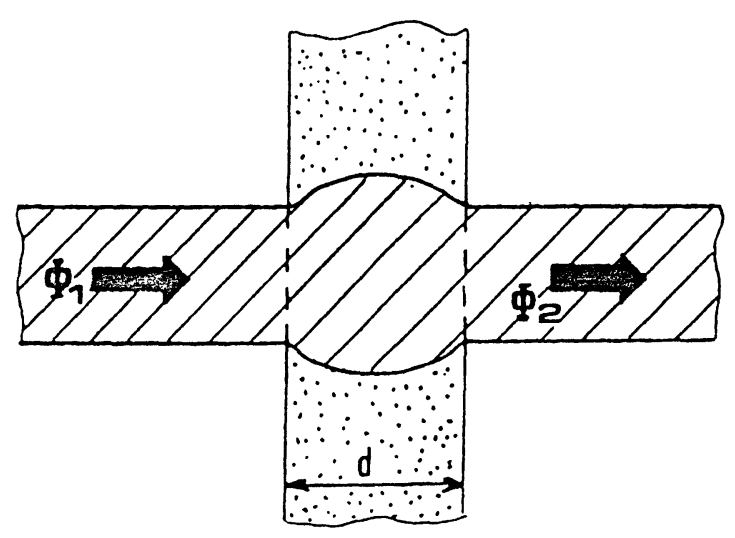

Fig. 14. - Epanouissement des lignes de champ.

[Spread of the magnetic field.] 
Tableau I. - Elément $Z_{1}$ en fonction de la fréquence pour $d=2 \mathrm{~mm}$.

[ $Z_{1}$ element with frequency variation in the case of $d=2 \mathrm{~mm}$.]

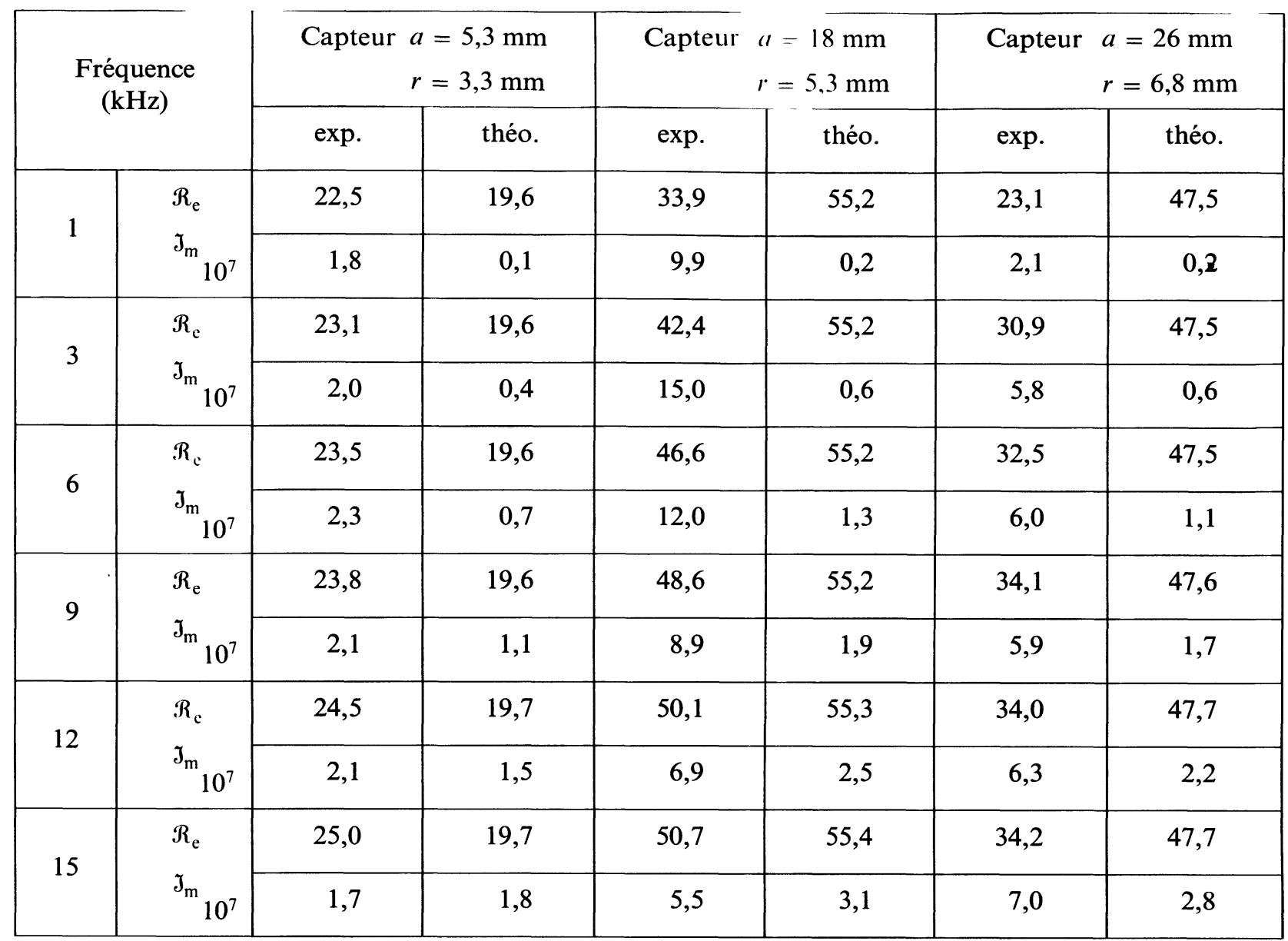

Le tableau I regroupe les résultats expérimentaux et théoriques des parties réelles et imaginaires de l'élément $Z_{1}$ obtenues en fonction de la fréquence, pour plusieurs configurations de capteurs.

Le calcul donne un ordre de grandeur acceptable pour les valeurs de la partie réelle de $Z_{1}$, mais semble par contre plus incertain sur les valeurs de la partie imaginaire. L'écart constaté provient non seulement des erreurs de mesure possibles mais aussi de la modélisation des lignes de fuite par un terme linéique parallèle réel. Pour remédier à ceci, il au ra1 arre in ervenir un erme para e e comp ex qui alourdirait considérablement les calculs.

La partie imaginaire de $Z_{1}$ étant faible devant sa partie réelle, un tel remaniement du modèle n'apporterait pas une amélioration importante à l'obtention de la reluctance ramenée à l'entrée.

Le paragraphe suivant est consacré à l'exploitation $\mathrm{du}$ modèle pour quantifier les variations de perméance vue par le capteur lors d'un déplacement de la cible, et donne accès aux grandeurs « dynamique " et " phase optimale ". Remarquons que ces quantités sont obtenues à partir de la différence entre deux perméances, ce qui permet de s'affranchir des éléments parallèles $Y_{\|}$et $Y_{1}$ présents à l'entrée du schéma équivalent.

4.3 Utilisation DU MODÈLE. - La figure 15 présente, pour une épaisseur de paroi de $2 \mathrm{~mm}$, l'évolution du terme $\Delta G$ (module et phase) en fonction du rapport $r / a$ pour les trois capteurs précédents. Les courbes sont paramétrées en fréquence.

On obtient sur ces courbes un maximum de dynamique lorsque le rayon cylindrique des pôles $\mathrm{du}$ c minimise le terme série $Y_{0}$ car elle offre une surface plus vaste au passage du flux, et augmente la longueur des lignes de courants susceptibles de s'établir autour des tubes de flux. Cette remarque est d'ailleurs également valable pour des capteurs de dimensions plus importantes, à épaisseur de paroi donnée. Par contre ce maximum s'atténue comme prévu lorsque la fréquence croît, les courants de Foucault atténuant davantage le flux mesuré.

On constate également l'augmentation de la phase optimale avec la croissance du rapport $r / a$ car il se 

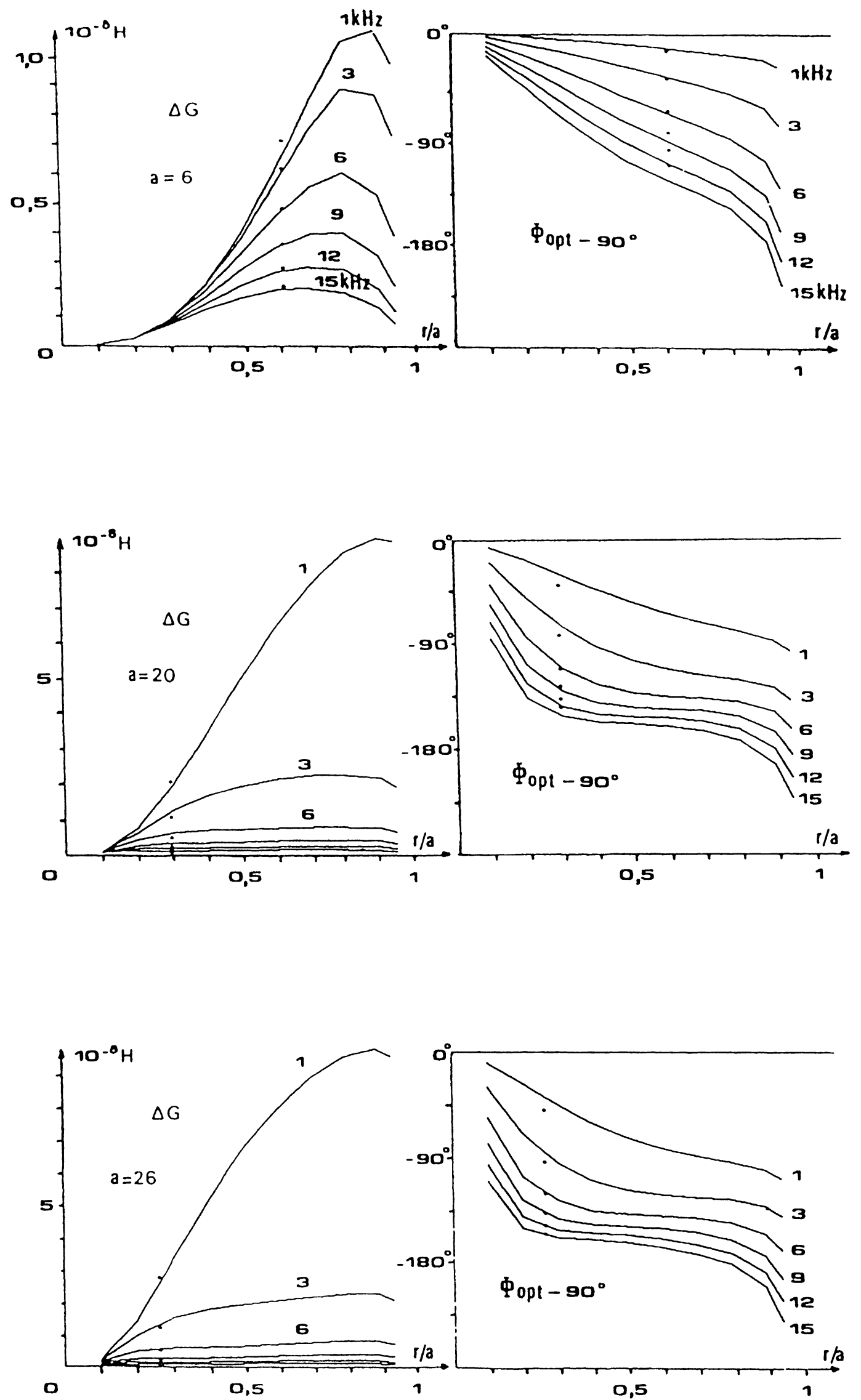

Fig. 15. - Dynamique et phase optimale en fonction de $r / a$.

[Dynamic range and optimal phase versus $r / a$ ratio.] 
crée une concentration accrue des courants induits dans une section devenue plus faible entre les pôles du capteur.

Nous avons reporté sur ces courbes théoriques les points correspondant aux mesures effectuées avec les différents capteurs, pour chaque épaisseur de paroi ; on constate une bonne concordance entre les données théoriques et les résultats expérimentaux. Cependant, lorsque l'épaisseur de la paroi ne peut être négligée vis-à-vis de l'écartement des pôles, il est nécessaire de corriger légèrement la valeur de $a$ et $r$ pour tenir compte de la géométrie réelle des lignes de champ dont on a vu qu'elles s'épanouissent plus volontiers vers l'extérieur du capteur (le rapport $r / a$ initial est conservé).

Par exemple, en ce qui concerne les courbes établies figure 15, nous avons choisi $a=6 \mathrm{~mm}$ pour une dimension réelle de $5,3 \mathrm{~mm}$ dans le premier cas, $20 \mathrm{~mm}$ au lieu de $18 \mathrm{~mm}$ dans le deuxième, et nous avons conservé la dimension réelle du troisième. La modification maximale apportée à ce paramètre est de l'ordre de $13 \%$ dans le premier cas, ce qui paraît raisonnable compte tenu de l'épaisseur déjà importante de la paroi $(2 \mathrm{~mm})$.

\section{Conclusion.}

Les aspects théoriques et expérimentaux abordés dans cet article démontrent la faisabilité d'un système de mesure de déplacements, conçu pour fonctionner à travers une paroi et ainsi préserver l'étanchéité d'un milieu soumis à des contraintes particulières (hautes pressions, milieu inflammable ou toxique, etc.).

Un modèle physique original de ce capteur permet de cerner convenablement le problème et de prédéterminer assez précisément la géométrie et les performances du système, lorsque la nature et l'épaisseur de la paroi protectrice sont connues. Un faible ajustement des paramètres de ce modèle autorise l'obtention d'un bon compromis entre la précision et la complexité des calculs.

Ces travaux permettent de compléter les notions théoriques et technologiques précédemment développées au sein de notre laboratoire, concernant l'utilisation de capteurs inductifs en analyse de profil et en mesure d'épaisseur sans contact, et contribuent à élargir le champ des applications industrielles réservées à ce type de capteurs.

\section{Remerciements.}

Ce travail a bénéficié du soutien de l'entreprise Technicatome. Les auteurs de cet article tiennent à remercier particulièrement Monsieur $\mathbf{M}$. Plantevin pour sa fructueuse collaboration.

\section{References}

[1] Clergeot H., Placko D., Monteil F., Flexible eddy current sensors for industrial applications, Proceedings Rovisec 4, London (October 84) pp. 115-122.

[2] JACOB D., Principe de la mesure simultanée de distance et d'épaisseur de dépôts métalliques par capteurs à courants de Foucault : conception et réalisation d'un dispositif « Thèse de Docteur en Sciences ", Université Paris-Sud (juin 1988).

[3] Placko D., Clergeot H., Santander E., Physical modelling of an eddy current sensor designed for real time distance and thickness measurement in galvanization industry, IEEE Trans. Magn. 25 (1989) 2861-2863.

[4] Brevet en cours de dépôt $n^{\circ} 8916556$, Société Technicatome, 14 décembre 1989 : Placko D., PlanTEVIN M., Capteur inductif et dispositif de mesure du déplacement d'un organe mobile.
[5] Placko D., Dispositif d'analyse de profil utilisant des capteurs à courants de Foucault, Thèse de $3^{\mathrm{e}}$ cycle, Orsay (avril 1984).

[6] Stratton J. A., Théorie de l'Electromagnétisme (Dunod, Paris) 1961, p. 220.

[7] Clergeot H., Placko D., Detriche J. M., Electrical Proximity Sensors, Sensors and Sensors Systems for Advanced Robots, NATO ASI Ser. (Italy) 43 (1986) 295-308.

[8] Gardiol F., Hyperfréquences, Traité d'électronique et d'électrotechnique (Dunod, Paris) 1987, pp. 382-393.

[9] Morse and Feshbach, Methods of Theoretical Physics, Part II (McGraw-Hill Book Company) 1953, p. 1259.

[10] Gardiol F., Electromagnétisme, Traité d'électronique et d'électrotechnique (Dunod, Paris) 1987, p. 171-173. 